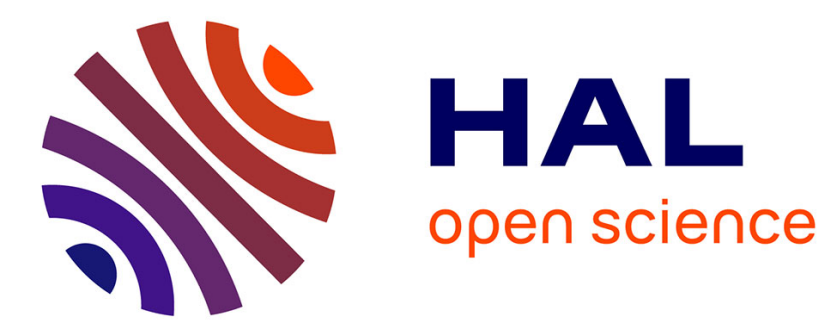

\title{
Benchmarking the $(1,4)$-CMA-ES With Mirrored Sampling and Sequential Selection on the Noisy BBOB-2010 Testbed
}

Anne Auger, Dimo Brockhoff, Nikolaus Hansen

\section{- To cite this version:}

Anne Auger, Dimo Brockhoff, Nikolaus Hansen. Benchmarking the (1,4)-CMA-ES With Mirrored Sampling and Sequential Selection on the Noisy BBOB-2010 Testbed. GECCO workshop on BlackBox Optimization Benchmarking (BBOB'2010), Jul 2010, Portland, OR, United States. pp.1625-1632, 10.1145/1830761.1830782 . inria-00502441

\section{HAL Id: inria-00502441 \\ https://hal.inria.fr/inria-00502441}

Submitted on 14 Jul 2010

HAL is a multi-disciplinary open access archive for the deposit and dissemination of scientific research documents, whether they are published or not. The documents may come from teaching and research institutions in France or abroad, or from public or private research centers.
L'archive ouverte pluridisciplinaire HAL, est destinée au dépôt et à la diffusion de documents scientifiques de niveau recherche, publiés ou non, émanant des établissements d'enseignement et de recherche français ou étrangers, des laboratoires publics ou privés. 


\title{
Benchmarking the $(1,4)-C M A-E S$ With Mirrored Sampling and Sequential Selection on the Noisy BBOB-2010 Testbed
}

\author{
[Black-Box Optimization Benchmarking Workshop]
}

\author{
Anne Auger, Dimo Brockhoff, and Nikolaus Hansen \\ Projet TAO, INRIA Saclay-lle-de-France \\ LRI, Bât 490, Univ. Paris-Sud \\ 91405 Orsay Cedex, France \\ firstname.lastname@inria.fr
}

\begin{abstract}
The Covariance-Matrix-Adaptation Evolution-Strategy (CMA-ES) is a robust stochastic search algorithm for optimizing functions defined on a continuous search space $\mathbb{R}^{D}$. Recently, mirrored samples and sequential selection have been introduced within CMA-ES to improve its local search performances. In this paper, we benchmark the $\left(1,4_{\mathrm{m}}^{\mathrm{s}}\right)$ CMA-ES which implements mirrored samples and sequential selection on the BBOB-2010 noisy testbed. Independent restarts are conducted until a maximal number of $10^{4} \mathrm{D}$ function evaluations is reached.

Although the tested $\left(1,4_{\mathrm{m}}^{\mathrm{s}}\right)$-CMA-ES is only a local search strategy, it solves 8 of the noisy BBOB-2010 functions in $20 \mathrm{D}$ and 9 of them in 5D for a target of $10^{-8}$. There is also one additional function in 20D and 5 additional functions in $5 \mathrm{D}$ where a successful run for at least one of the 15 instances can be reported. Moreover, on 7 of the 8 functions that are solved by the $\left(1,4_{\mathrm{m}}^{\mathrm{s}}\right)$-CMA-ES in $20 \mathrm{D}$, we see a large improvement over the best algorithm of the BBOB-2009 benchmarking for the corresponding functions - ranging from an $37 \%$ improvement on the sphere with moderate Cauchy noise to a speed-up by a factor of about 3 on the Gallagher function with Cauchy noise.
\end{abstract}

\section{Categories and Subject Descriptors}

G.1.6 [Numerical Analysis]: Optimization-global optimization, unconstrained optimization; F.2.1 [Analysis of Algorithms and Problem Complexity]: Numerical Algorithms and Problems

\section{General Terms}

Algorithms

\section{Keywords}

Benchmarking, Black-box optimization

(C)ACM, 2010. This is the authors' version of the work. It is posted here by permission of ACM for your personal use. Not for redistribution. The definitive version was published at GECCO'10, July 7-11, 2010, Portland, OR, USA. http://doi.acm.org/10.1145/1830761.1830782

\section{INTRODUCTION}

Evolution Strategies (ESs) are stochastic search algorithms designed to minimize ${ }^{1}$ objective functions, $f$, mapping a continuous search space $\mathbb{R}^{D}$ into $\mathbb{R}$. Among ESs, the Covariance-Matrix-Adaptation Evolution-Strategy (CMA-ES) is now a well recognized algorithm. In the standard $(\mu /$ $\left.\mu_{w}, \lambda\right)$-CMA-ES $[18,25]$, at each iteration step $n$, a set of $\lambda$ candidate solutions is created by sampling random vectors distributed according to a multivariate normal distribution with mean vector zero and covariance matrix $\boldsymbol{C}_{n}$. Those $\lambda$ random vectors denoted $\left(\mathcal{N}_{i}\left(\mathbf{0}, \boldsymbol{C}_{n}\right)\right)_{1 \leq i \leq \lambda}$ are multiplied by a strictly possitive factor, the step-size $\sigma_{n}$, and added to the current solution $\boldsymbol{X}_{n}$ to constitute the offspring $\boldsymbol{X}_{n}^{i}=\boldsymbol{X}_{n}+\sigma_{n} \mathcal{N}_{i}\left(\mathbf{0}, \boldsymbol{C}_{n}\right)$. After evaluation of the $\lambda$ offspring, the $\mu$ best, i.e., the ones having the smallest objective function values, are selected. The current solution is updated to the average value of the $\mu$ best solutions: $\boldsymbol{X}_{n+1}=$ $\sum_{i=1}^{\mu} w_{i} \boldsymbol{X}_{n}^{i: \lambda}$, where $w_{1} \geq \ldots \geq w_{\mu}$ and $\sum_{i=1}^{\mu} w_{i}=1$ and $\boldsymbol{X}_{n}^{i: \lambda}$ denotes the $i$-th best offspring. Covariance matrix and step-size are then updated using solely the information given by the ranking of the offspring. Though originally designed to be a robust local search $[26]$, the $\left(\mu / \mu_{w}, \lambda\right)$-CMAES turns out to be also effective for multi-modal functions provided a large enough population size $\mu=\lambda / 2$ is chosen [25]. An automatic way to increase the probability to converge on multi-modal functions consists in applying restarts with a successively increasing population size. The strategy is then called IPOP-CMA-ES [13]. However, deceptive functions were constructed for the IPOP-CMA-ES [27, 23]. The BBOB function $f_{24}$ presents, in a highly rugged landscape, on the larger scale an attraction region for the global optimum which is smaller than the one for the local optimum. For that reason, the BIPOP-CMA-ES, combining restarts with increasing population size as well as with a fixed small population size, was proposed [19]. For the large budgets that are needed for most multi-modal problems, the BIPOP-CMA-ES performed overall best for the BBOB-2009 workshop [22].

While BIPOP-CMA-ES was benchmarked, the local search $(1+1)-C M A-E S$ was as well tested $[14,15]$. Surprisingly, the $(1+1)$-variant of CMA-ES could outperform the BIPOPCMA-ES algorithm by a significant factor on the Gallagher functions $f_{21}$ and $f_{22}[11]$. On $f_{21}$, the $(1+1)$-CMA-ES is 8.2 times (resp. 68.7 times) faster than the BIPOP-CMA-

\footnotetext{
${ }^{1}$ We assume without loss of generality minimization since maximizing $f$ amounts to minimize $-f$.
} 
ES in dimension 20 (resp. 40); for $f_{22}$, the (1+1)-CMA-ES is 37 times faster than the BIPOP-CMA-ES in 20D and is able to solve the problem in 40D which the BIPOP-CMAES does not allow. However, one major drawback of elitist selection, used in the (1+1)-CMA-ES, is the complete lack of robustness in presence of noise [15].

Motivated by the surprisingly large improvement over the BIPOP-CMA, new non-elitist local search ESs have been proposed [6]. Those $(1, \lambda)$-ESs combine a derandomization technique by means of mirrored samples with a sequential selection scheme. Mirrored samples replace the independent random vectors used for the offspring. Instead of the $\lambda$ independent random vectors, only $\lambda / 2$ (assuming $\lambda$ is even) independent samples are generated $\left(\mathcal{N}_{2 i-1}\left(\mathbf{0}, \boldsymbol{C}_{n}\right)\right)_{1 \leq i \leq \lambda / 2}$. The other $\lambda / 2$ samples are replaced by the already generated samples multiplied by -1 , i.e., $\mathcal{N}_{2 i}\left(\mathbf{0}, \boldsymbol{C}_{n}\right)=-\mathcal{N}_{2 i-1}\left(\mathbf{0}, \boldsymbol{C}_{n}\right)$ for all $1 \leq i \leq \lambda / 2$. The resulting offspring are two by two symmetrical or mirrored with respect to $\boldsymbol{X}_{n}$. Sequential selection consists in performing the evaluations of the $\lambda$ offspring sequentially and comparing after each evaluation the offspring solution $\boldsymbol{X}_{n}^{i}$ with the current solution $\boldsymbol{X}_{n}$. If $f\left(\boldsymbol{X}_{n}^{i}\right) \leq f\left(\boldsymbol{X}_{n}\right)$, the sequence of evaluations is stopped and $\boldsymbol{X}_{n+1}=\boldsymbol{X}_{n}^{i}$, saving thus the remaining offspring evaluations.

The impact of mirrored samples and sequential selection has been investigated on the BBOB-2010 for the (1,2)-CMA$\operatorname{ES}[2,3,7,8]$ and for the (1,4)-CMA-ES $[4,5,9,10]$. The purpose of this paper is to present the results of one of those strategies tested, namely the (1,4)-CMA-ES with mirrored samples and sequential selection on the BBOB-2010 noisy testbed. Since the algorithm tested is a local search strategy, we do not expect that it will perform well on the whole testbed but rather want to see whether the strategy can bring some improvements over last year's results on certain functions.

The tested algorithm $\left(1,4_{\mathrm{m}}^{\mathrm{s}}\right)$-CMA-ES as well as the CPU timing experiments are described in a complementing paper in the same proceedings [1].

\section{RESULTS AND DISCUSSION}

Results from experiments according to [21] on the benchmark functions given in $[16,24]$ are presented in Figures 1, 2 and 3 and in Tables 1,2 and 3.

Although the tested $\left(1,4_{\mathrm{m}}^{\mathrm{s}}\right)$-CMA-ES is only a local search strategy, it solves 8 of the noisy BBOB-2010 functions in 20D and 9 of them in $5 \mathrm{D}$ for a target of $10^{-8}$. In addition, there is one function in 20D and 5 functions in $5 \mathrm{D}$ where a successful run for at least one of the 15 instances can be reported. In the light of this result, it is worth to mention that the noisy test functions in the BBOB-2009 testbed ${ }^{2}$ have not been solved as successfully as the noiseless ones: 9 out of the 30 functions could not been solved by any algorithm or solely by the BIPOP-CMA-ES of [20], see [12] for details about the BBOB-2009 results. Moreover, on 7 of the 8 functions that are solved by $\left(1,4_{\mathrm{m}}^{\mathrm{s}}\right)$-CMA-ES in $20 \mathrm{D}$, we see even an improvement over the function-wise best algorithm of the BBOB-2009 benchmarking which we detail below.

On the sphere function with moderate noise $\left(f_{103}\right)$, the $\left(1,4_{\mathrm{m}}^{\mathrm{s}}\right)$-CMA-ES is about $35 \%$ better than the best algo-

\footnotetext{
${ }^{2}$ These are the same functions than in the BBOB-2010 testbed with the only difference that instead of 15 instances per function, three independent runs were performed on 5 different instances within BBOB-2009.
}

rithm for this function in $20 \mathrm{D}$ and for a target of $10^{-7}$. For all other targets, the $\left(1,4_{\mathrm{m}}^{\mathrm{s}}\right)$-CMA-ES also outperforms the best algorithm for this function. The best algorithm of the BBOB-2009 benchmarking on this function turns out to be the IPOP-SEP-CMA-ES [28] for small, i.e., difficult targets.

On the Rosenbrock function with moderate Cauchy noise $\left(f_{106}\right)$, the $\left(1,4_{\mathrm{m}}^{\mathrm{s}}\right)$-CMA-ES outperforms the best BBOB2009 algorithm for this function in all dimensions $(2 \mathrm{D}, 3 \mathrm{D}$, 5D, 10D, and 20D) and for all small targets which also here is the IPOP-SEP-CMA-ES [28]. The expected running time of the $\left(1,4_{\mathrm{m}}^{\mathrm{s}}\right)$-CMA-ES is thereby about $40 \%$ smaller than for the IPOP-SEP-CMA-ES for a target value of $10^{-7}$ in $20 \mathrm{D}$.

Also the sphere function with Cauchy noise $\left(f_{109}\right)$ is solved faster by the $\left(1,4_{\mathrm{m}}^{\mathrm{s}}\right)$-CMA-ES than the best algorithm of the BBOB-2009 benchmarking on this function in 5D, 10D, and $20 \mathrm{D}$ where the improvement is about $50 \%$ in $20 \mathrm{D}$ and where both the IPOP-SEP-CMA-ES and the BIPOP-CMA-ES [20] are the best algorithms of BBOB-2009 on this function.

A $50 \%$ improvement can also be seen on the Rosenbrock function with Cauchy noise $\left(f_{112}\right)$ in $20 \mathrm{D}$ and for small targets. Better results than the IPOP-SEP-CMA-ES, the best algorithm for this function in BBOB-2009, are also obtained in $2 \mathrm{D}, 3 \mathrm{D}, 5 \mathrm{D}$ and $10 \mathrm{D}$ here.

The improvement over the best algorithm of BBOB-2009 on the ellipsoid function with Cauchy noise $\left(f_{118}\right)$ is about $40 \%$ in $20 \mathrm{D}$ (for all small targets). The best algorithms in BBOB-2009 on this function are the IPOP-SEP-CMA-ES (for a target value of $10^{-7}$ ) as well as the algorithm VNC [17] (for all target values). Better results on $f_{118}$ than the best algorithm of BBOB-2009 are also obtained in 2D, 3D, $5 \mathrm{D}$, and 10D.

On the sum of different powers function comprising Cauchy noise $\left(f_{121}\right)$, the $\left(1,4_{\mathrm{m}}^{\mathrm{s}}\right)$-CMA-ES shows expected running times that are at least $48 \%$ lower than the best algorithm of BBOB-2009 on this function in 20D and for small target values. Better results than the best BBOB-2009 algorithm on this function in $2 \mathrm{D}, 3 \mathrm{D}, 5 \mathrm{D}$, and $10 \mathrm{D}$ can also be reported.

Last, also on the Gallagher function with Cauchy noise $\left(f_{130}\right)$, an improvement over the best algorithm of BBOB2009 on this function can be seen in the results. Here, we see the largest impact of the $\left(1,4_{\mathrm{m}}^{\mathrm{s}}\right)$-CMA-ES, where the expected running times are, in $20 \mathrm{D}$ and for several small targets, by a factor of about 3 smaller than the ones of the best algorithm of BBOB-2009 on this function. Also the results in $2 \mathrm{D}, 3 \mathrm{D}, 5 \mathrm{D}$, and $10 \mathrm{D}$ are better for the $\left(1,4_{\mathrm{m}}^{\mathrm{s}}\right)$-CMA-ES where the improvement factor only slightly differs in $3 \mathrm{D}$, $5 \mathrm{D}$, and 10D. At least for small targets and dimension $\geq 5$, the IPOP-SEP-CMA-ES is here also the best algorithm of BBOB-2009.

Note that all functions, where an improvement over the best algorithm of the BBOB-2009 benchmarking can be reported, comprise a Cauchy noise. Cauchy noise is only sampled $20 \%$ of the time, such that it is enough to be robust to postive and negative outliers for solving those functions. For the other noise types, most probably a larger population size or another method to cope with the noise is needed. Furthermore, the maximum number of function evaluations was chosen quite small for solving the more difficult noise types up to the final target value. 

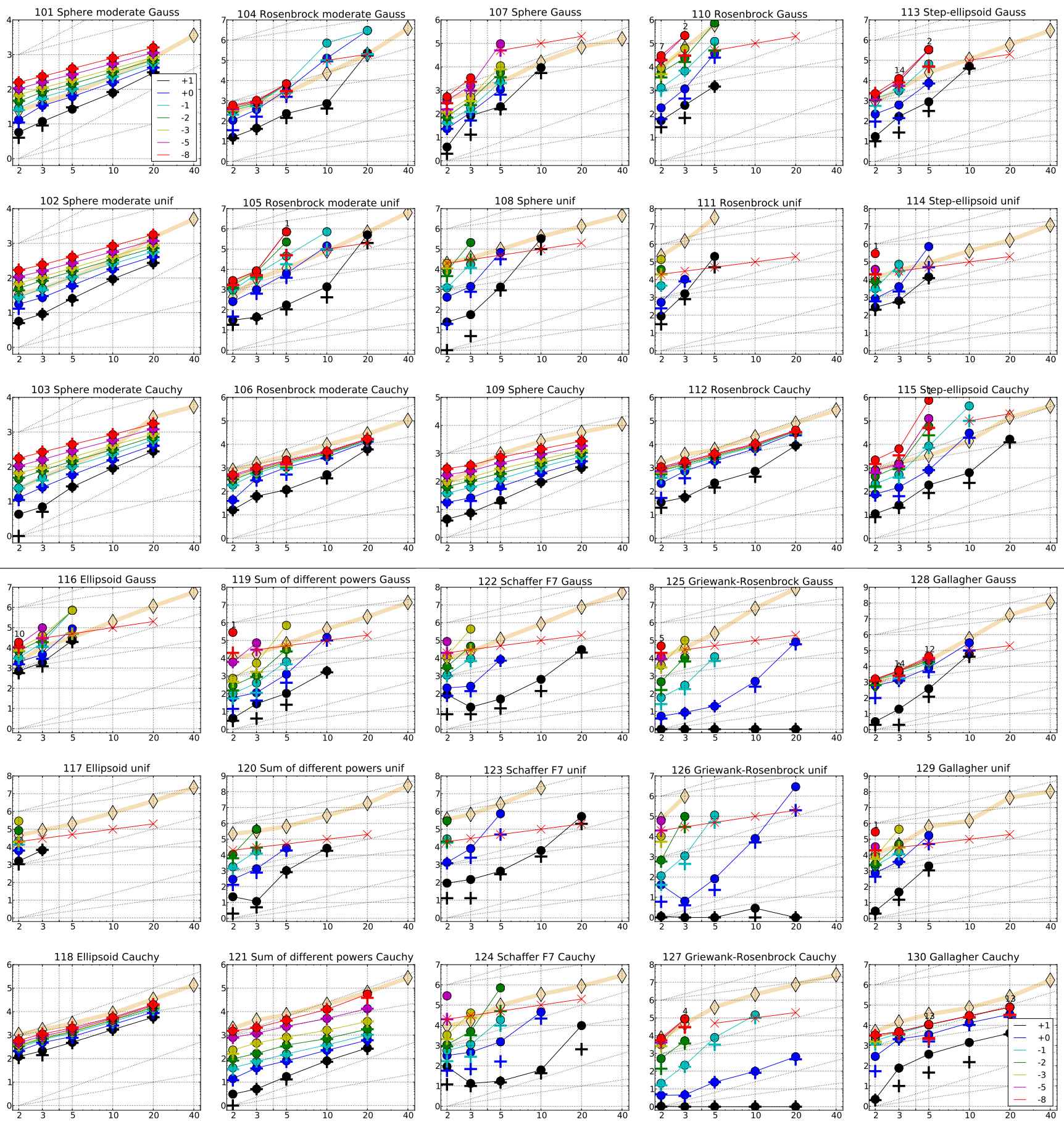

Figure 1: Expected Running Time (ERT, - ) to reach $f_{\mathrm{opt}}+\Delta f$ and median number of $f$-evaluations from successful trials $(+)$, for $\Delta f=10^{\{+1,0,-1,-2,-3,-5,-8\}}$ (the exponent is given in the legend of $f_{101}$ and $f_{130}$ ) versus dimension in log-log presentation. For each function and dimension, $\operatorname{ERT}(\Delta f)$ equals to \#FEs $(\Delta f)$ divided by the number of successful trials, where a trial is successful if $f_{\mathrm{opt}}+\Delta f$ was surpassed. The \#FEs $(\Delta f)$ are the total number (sum) of $f$-evaluations while $f_{\mathrm{opt}}+\Delta f$ was not surpassed in the trial, from all (successful and unsuccessful) trials, and $f_{\text {opt }}$ is the optimal function value. Crosses $(\times)$ indicate the total number of $f$-evaluations, \#FEs $(-\infty)$, divided by the number of trials. Numbers above ERT-symbols indicate the number of successful trials. Y-axis annotations are decimal logarithms. The thick light line with diamonds shows the single best results from BBOB-2009 for $\Delta f=10^{-8}$. Additional grid lines show linear and quadratic scaling. 

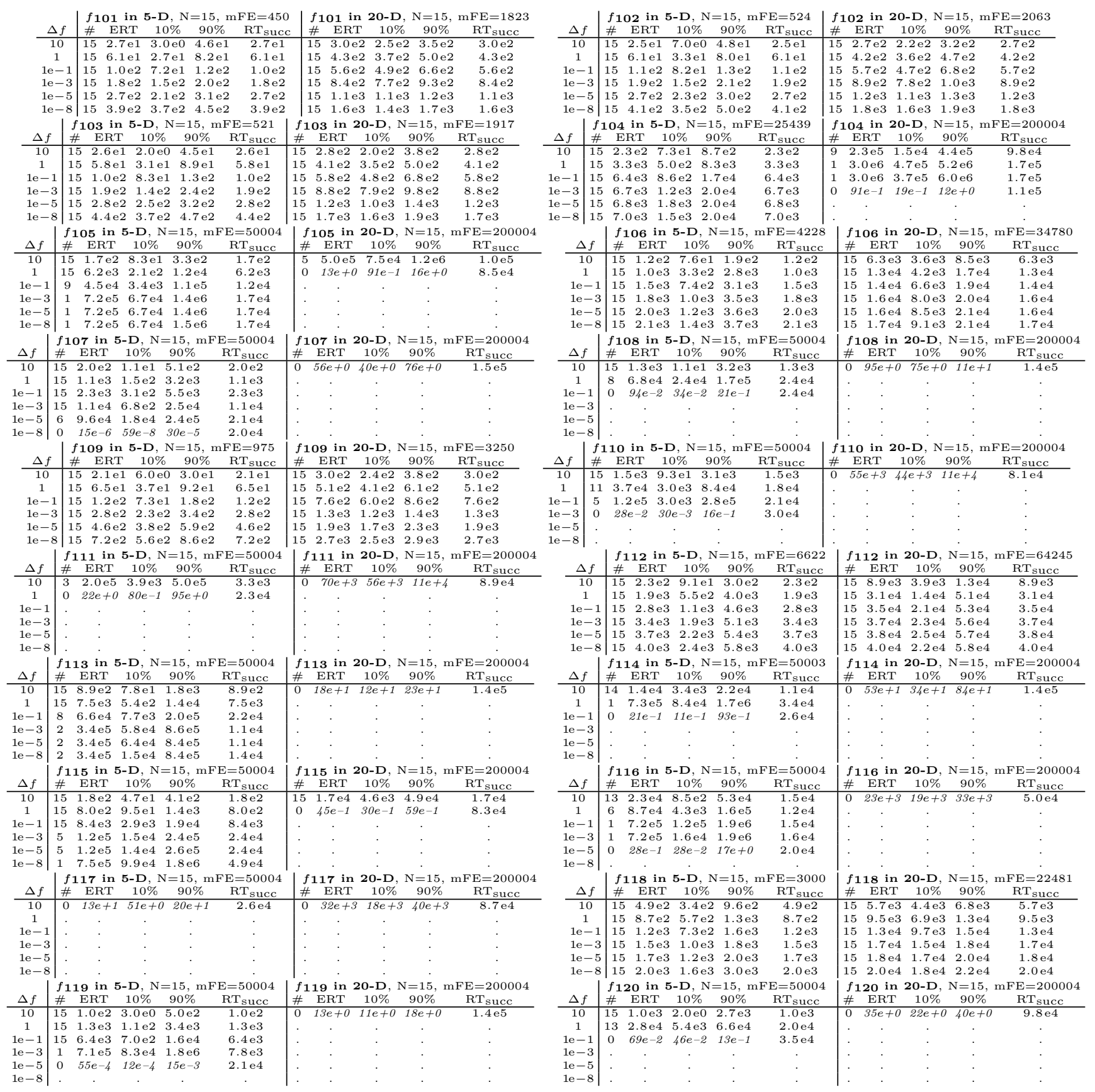

Table 1: Shown are, for functions $f_{101}-f_{120}$ and for a given target difference to the optimal function value $\Delta f$ : the number of successful trials (\#); the expected running time to surpass $f_{\mathrm{opt}}+\Delta f$ (ERT, see Figure 1); the $10 \%$-tile and $90 \%$-tile of the bootstrap distribution of ERT; the average number of function evaluations in successful trials or, if none was successful, as last entry the median number of function evaluations to reach the best function value $\left(\mathbf{R T}_{\text {succ }}\right)$. If $f_{\mathrm{opt}}+\Delta f$ was never reached, figures in italics denote the best achieved $\Delta f$-value of the median trial and the $10 \%$ and $90 \%$-tile trial. Furthermore, $\mathbf{N}$ denotes the number of trials, and $\mathrm{mFE}$ denotes the maximum of number of function evaluations executed in one trial. See Figure 1 for the names of functions. 

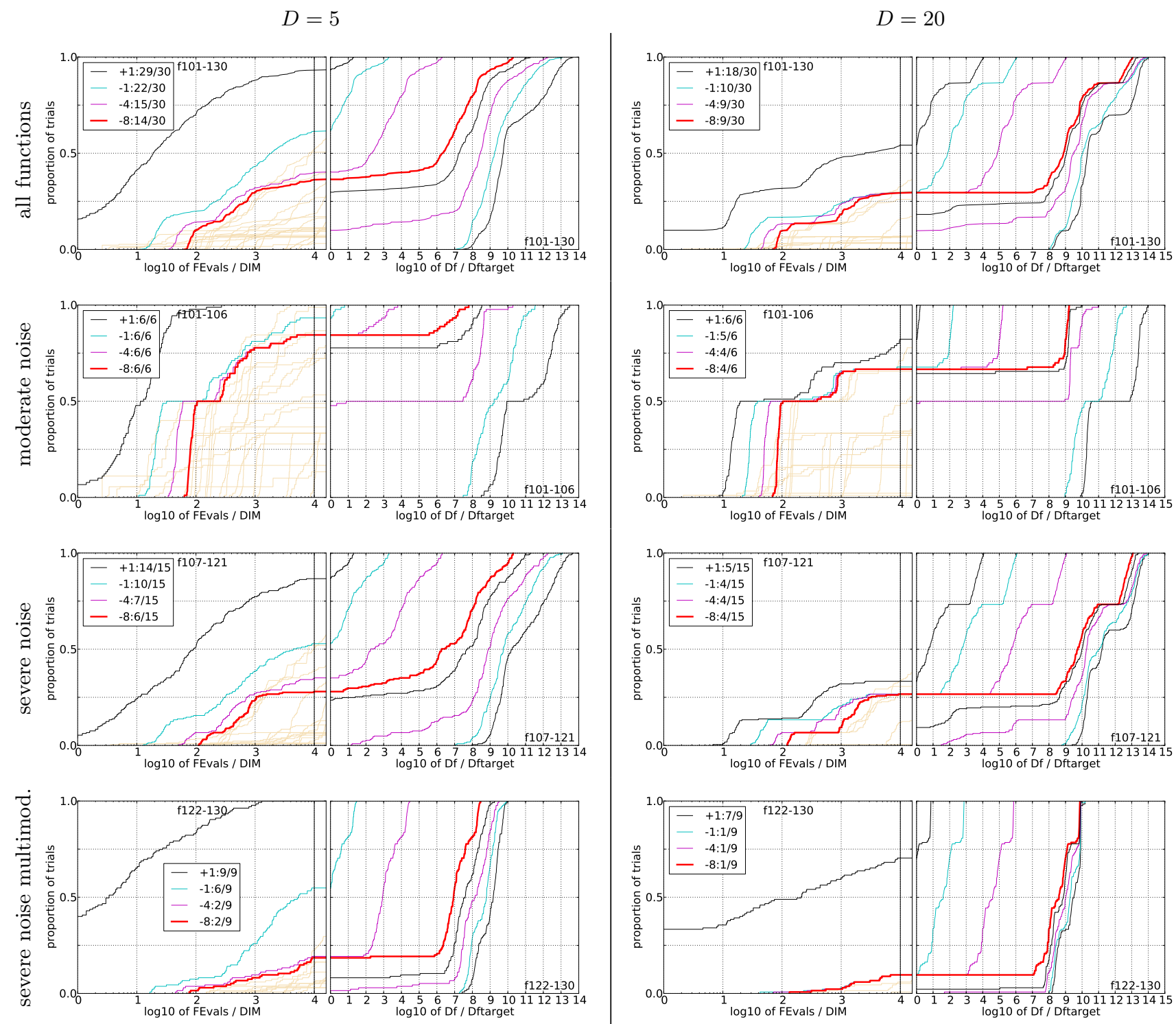

Figure 2: Empirical cumulative distribution functions (ECDFs), plotting the fraction of trials versus running time (left subplots) or versus $\Delta f$ (right subplots). The thick red line represents the best achieved results. Left subplots: ECDF of the running time (number of function evaluations), divided by search space dimension $D$, to fall below $f_{\text {opt }}+\Delta f$ with $\Delta f=10^{k}$, where $k$ is the first value in the legend. Right subplots: ECDF of the best achieved $\Delta f$ divided by $10^{k}$ (upper left lines in continuation of the left subplot), and best achieved $\Delta f$ divided by $10^{-8}$ for running times of $D, 10 D, 100 \mathrm{D} \ldots$ function evaluations (from right to left cycling blackcyan-magenta). The legends indicate the number of functions that were solved in at least one trial. FEvals denotes number of function evaluations, $D$ and DIM denote search space dimension, and $\Delta f$ and Df denote the difference to the optimal function value. Light brown lines in the background show ECDFs for target value $10^{-8}$ of all algorithms benchmarked during BBOB-2009. 

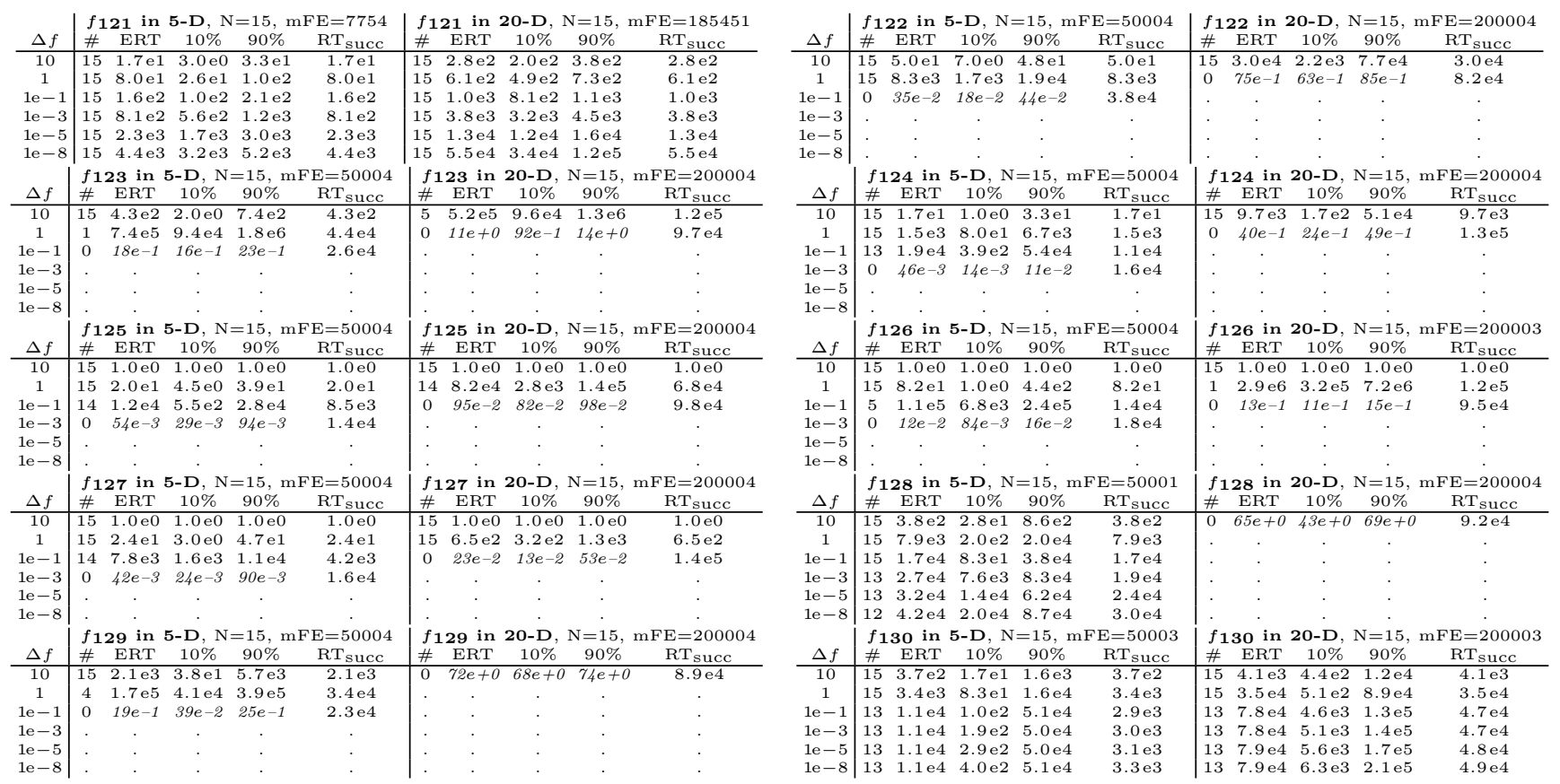

Table 2: Shown are, for functions $f_{121}-f_{130}$ and for a given target difference to the optimal function value $\Delta f$ : the number of successful trials (\#); the expected running time to surpass $f_{\mathrm{opt}}+\Delta f$ (ERT, see Figure 1); the $10 \%$-tile and $90 \%$-tile of the bootstrap distribution of ERT; the average number of function evaluations in successful trials or, if none was successful, as last entry the median number of function evaluations to reach the best function value $\left(\mathbf{R T}_{\text {succ }}\right)$. If $f_{\mathrm{opt}}+\Delta f$ was never reached, figures in italics denote the best achieved $\Delta f$-value of the median trial and the $10 \%$ and $\mathbf{9 0 \%}$-tile trial. Furthermore, $\mathbf{N}$ denotes the number of trials, and $\mathrm{mFE}$ denotes the maximum of number of function evaluations executed in one trial. See Figure 1 for the names of functions.

\section{Acknowledgments}

This work receives support by the French national research agency (ANR) within the SYSCOMM project ANR-08-SYSC017 and within the COSINUS project ANR-08-COSI-007-12.

\section{REFERENCES}

[1] A. Auger, D. Brockhoff, and N. Hansen. Benchmarking the $(1,4)$-CMA-ES with mirrored sampling and sequential selection on the noiseless BBOB-2010 testbed. In GECCO (Companion), 2010.

[2] A. Auger, D. Brockhoff, and N. Hansen. Investigating the impact of sequential selection in the (1,2)-CMA-ES on the noiseless BBOB-2010 testbed. In GECCO (Companion), 2010.

[3] A. Auger, D. Brockhoff, and N. Hansen. Investigating the impact of sequential selection in the (1,2)-CMA-ES on the noisy BBOB-2010 testbed. In GECCO (Companion), 2010.

[4] A. Auger, D. Brockhoff, and N. Hansen. Investigating the impact of sequential selection in the $(1,4)$-CMA-ES on the noiseless BBOB-2010 testbed. In GECCO (Companion), 2010.

[5] A. Auger, D. Brockhoff, and N. Hansen. Investigating the impact of sequential selection in the $(1,4)$-CMA-ES on the noisy BBOB-2010 testbed. In GECCO (Companion), 2010.

[6] A. Auger, D. Brockhoff, and N. Hansen. Mirrored sampling and sequential selection for evolution
Table 3: ERT loss ratio (see Figure 3) compared to the respective best result from $\mathrm{BBOB-2009}$ for budgets given in the first column. The last row $R L_{U S} / D$ gives the number of function evaluations in unsuccessful runs divided by dimension. Shown are the smallest, $10 \%$-ile, $25 \%$-ile, $50 \%$-ile, $75 \%$-ile and $90 \%$ ile value (smaller values are better).

\begin{tabular}{|c|c|c|c|c|c|c|}
\hline & \multicolumn{6}{|c|}{$\boldsymbol{f} \mathbf{1 0 1}-\boldsymbol{f} \mathbf{1 3 0}$ in $\mathbf{5}-\mathrm{D}, \operatorname{maxFE} / \mathrm{D}=10000$} \\
\hline \#FEs/D & best & $10 \%$ & $25 \%$ & med & $75 \%$ & $90 \%$ \\
\hline 2 & 0.91 & 1.8 & 2.7 & 6.2 & 10 & 10 \\
\hline 10 & 0.94 & 1.4 & 1.9 & 5.2 & 36 & 50 \\
\hline 100 & 0.52 & 1.1 & 2.1 & 5.4 & 43 & $3.0 \mathrm{e} 2$ \\
\hline $1 \mathrm{e} 3$ & 0.60 & 0.67 & 3.8 & 5.8 & 26 & $2.5 \mathrm{e} 3$ \\
\hline $1 \mathrm{e} 4$ & 0.32 & 0.66 & 1.8 & 12 & 24 & $2.5 \mathrm{e} 4$ \\
\hline $\mathrm{RL}_{\mathrm{US}} / \mathrm{D}$ & $1 \mathrm{e} 4$ & $1 \mathrm{e} 4$ & $1 \mathrm{e} 4$ & $1 \mathrm{e} 4$ & $1 \mathrm{e} 4$ & $1 \mathrm{e} 4$ \\
\hline & \multicolumn{6}{|c|}{$\boldsymbol{f} \mathbf{1 0 1}-\boldsymbol{f} \mathbf{1 3 0}$ in $\mathbf{2 0}-\mathrm{D}, \operatorname{maxFE} / \mathrm{D}=10000$} \\
\hline$\# \mathrm{FEs} / \mathrm{D}$ & best & $10 \%$ & $25 \%$ & med & $75 \%$ & $90 \%$ \\
\hline 2 & 1.0 & 5.0 & 40 & 40 & 40 & 40 \\
\hline 10 & 1.0 & 3.0 & 4.7 & $1.6 \mathrm{e} 2$ & $2.0 \mathrm{e} 2$ & $2.0 \mathrm{e} 2$ \\
\hline 100 & 0.52 & 0.65 & 1.4 & $1.6 \mathrm{e} 2$ & $2.0 \mathrm{e} 3$ & $2.0 \mathrm{e} 3$ \\
\hline $1 \mathrm{e} 3$ & 0.27 & 0.46 & 1.2 & $3.7 \mathrm{e} 2$ & $2.0 \mathrm{e} 4$ & $2.0 \mathrm{e} 4$ \\
\hline $1 \mathrm{e} 4$ & 0.31 & 0.55 & 1.2 & $2.8 \mathrm{e} 2$ & $2.4 \mathrm{e} 3$ & $2.0 \mathrm{e} 5$ \\
\hline $1 \mathrm{e} 5$ & 0.31 & 0.55 & 1.2 & $1.8 \mathrm{e} 3$ & $4.7 \mathrm{e} 3$ & $2.0 \mathrm{e} 6$ \\
\hline $\mathrm{RL}_{\mathrm{US}} / \mathrm{D}$ & $1 \mathrm{e} 4$ & $1 \mathrm{e} 4$ & $1 \mathrm{e} 4$ & $1 \mathrm{e} 4$ & $1 \mathrm{e} 4$ & $1 \mathrm{e} 4$ \\
\hline
\end{tabular}



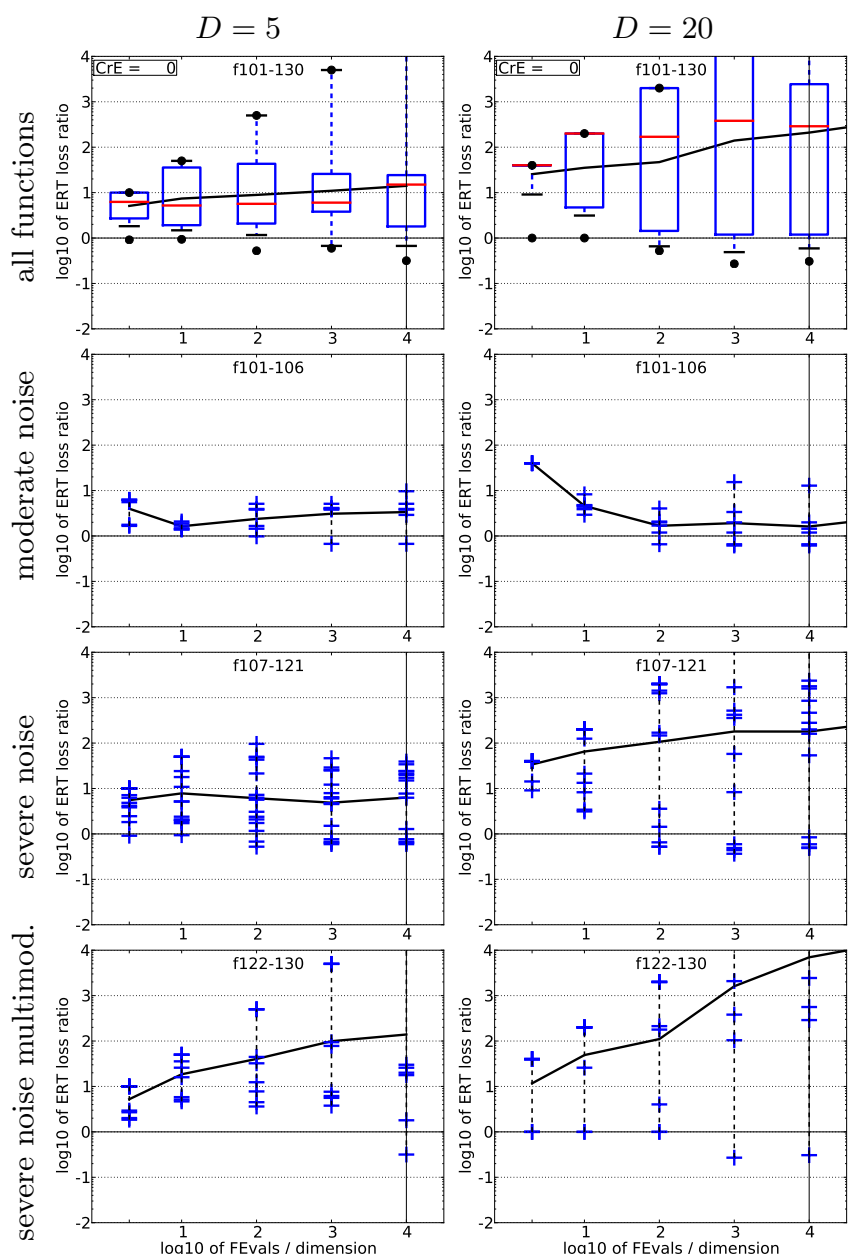

Figure 3: ERT loss ratio versus given budget FEvals. The target value $f_{\mathrm{t}}$ for ERT (see Figure 1) is the smallest (best) recorded function value such that $\operatorname{ERT}\left(f_{\mathrm{t}}\right) \leq$ FEvals for the presented algorithm. Shown is FEvals divided by the respective best $\operatorname{ERT}\left(f_{\mathrm{t}}\right)$ from BBOB-2009 for functions $f_{101}-f_{130}$ in 5-D and 20D. Each ERT is multiplied by $\exp (\mathrm{CrE})$ correcting for the parameter crafting effort. Line: geometric mean. Box-Whisker error bar: 25-75\%-ile with median (box), 10-90\%-ile (caps), and minimum and maximum ERT loss ratio (points). The vertical line gives the maximal number of function evaluations in this function subset.

strategies. Rapport de Recherche RR-7249, INRIA Saclay-Île-de-France, April 2010.

[7] A. Auger, D. Brockhoff, and N. Hansen. Mirrored variants of the $(1,2)$-CMA-ES compared on the noiseless BBOB-2010 testbed. In GECCO (Companion), 2010.

[8] A. Auger, D. Brockhoff, and N. Hansen. Mirrored variants of the $(1,2)$-CMA-ES compared on the noisy BBOB-2010 testbed. In GECCO (Companion), 2010.

[9] A. Auger, D. Brockhoff, and N. Hansen. Mirrored variants of the $(1,4)$-CMA-ES compared on the noiseless BBOB-2010 testbed. In GECCO (Companion), 2010.
[10] A. Auger, D. Brockhoff, and N. Hansen. Mirrored variants of the $(1,4)$-CMA-ES compared on the noisy BBOB-2010 testbed. In GECCO (Companion), 2010.

[11] A. Auger, S. Finck, N. Hansen, and R. Ros. BBOB 2009: Comparison tables of all algorithms on all noiseless functions. Technical Report RT-0383, INRIA, April 2010.

[12] A. Auger, S. Finck, N. Hansen, and R. Ros. BBOB 2009: Comparison tables of all algorithms on all noisy functions. Technical Report RT-0384, INRIA, 042010.

[13] A. Auger and N. Hansen. A restart CMA evolution strategy with increasing population size. In Proc. IEEE Congress On Evolutionary Computation, pages 1769-1776, 2005.

[14] A. Auger and N. Hansen. Benchmarking the (1+1)-CMA-ES on the BBOB-2009 function testbed. In Rothlauf [29], pages 2459-2466.

[15] A. Auger and N. Hansen. Benchmarking the (1+1)-CMA-ES on the BBOB-2009 noisy testbed. In Rothlauf [29], pages 2467-2472.

[16] S. Finck, N. Hansen, R. Ros, and A. Auger. Real-parameter black-box optimization benchmarking 2010: Presentation of the noisy functions. Technical Report 2009/21, Research Center PPE, 2010.

[17] C. García-Martínez and M. Lozano. A continuous variable neighbourhood search based on specialised EAs: application to the noisy BBO-benchmark 2009 testbed. In Rothlauf [29], pages 2367-2374.

[18] N. Hansen. The CMA evolution strategy: a comparing review. In J. Lozano, P. Larranaga, I. Inza, and E. Bengoetxea, editors, Towards a new evolutionary computation. Advances on estimation of distribution algorithms, pages 75-102. Springer, 2006.

[19] N. Hansen. Benchmarking a BI-population CMA-ES on the BBOB-2009 function testbed. In Rothlauf [29], pages 2389-2396.

[20] N. Hansen. Benchmarking a BI-population CMA-ES on the BBOB-2009 noisy testbed. In Rothlauf [29], pages 2397-2402.

[21] N. Hansen, A. Auger, S. Finck, and R. Ros. Real-parameter black-box optimization benchmarking 2010: Experimental setup. Technical Report RR-7215, INRIA, 2010.

[22] N. Hansen, A. Auger, R. Ros, S. Finck, and P. Pošík. Comparing results of 31 algorithms from the black-box optimization benchmarking BBOB-2009. In Workshop Proceedings of the Genetic and Evolutionary Computation Conference (GECCO 2010). ACM Press, 2010. to appear.

[23] N. Hansen, S. Finck, R. Ros, and A. Auger. Real-parameter black-box optimization benchmarking 2009: Noiseless functions definitions. Technical Report RR-6829, INRIA, 2009.

[24] N. Hansen, S. Finck, R. Ros, and A. Auger. Real-parameter black-box optimization benchmarking 2009: Noisy functions definitions. Technical Report RR-6869, INRIA, 2009. Updated February 2010.

[25] N. Hansen and S. Kern. Evaluating the CMA evolution strategy on multimodal test functions. In X. Yao et al., editors, Parallel Problem Solving from Nature PPSN VIII, volume 3242 of $L N C S$, pages 282-291. Springer, 2004. 
[26] N. Hansen and A. Ostermeier. Completely derandomized self-adaptation in evolution strategies. Evolutionary Computation, 9(2):159-195, 2001.

[27] M. Lunacek, D. Whitley, and A. Sutton. The impact of global structure on search. In Proceedings of the 10th international conference on Parallel Problem Solving from Nature, pages 498-507, Berlin, Heidelberg, 2008. Springer-Verlag.
[28] R. Ros. Benchmarking sep-CMA-ES on the BBOB-2009 noisy testbed. In Rothlauf [29], pages 2441-2446.

[29] F. Rothlauf, editor. Genetic and Evolutionary Computation Conference, GECCO 2009, Proceedings, Montreal, Québec, Canada, July 8-12, 2009, Companion Material. ACM, 2009. 